\title{
Duality Relations for a Class of a Multiobjective Fractional Programming Problem Involving Support Functions
}

\author{
Vandana1, Ramu Dubey², Deepmala ${ }^{3}$, Lakshmi Narayan Mishra ${ }^{4,5 *}$, Vishnu Narayan Mishra ${ }^{6}$ \\ ${ }^{1}$ Department of Management Studies, Indian Institute of Technology Madras, Chennai, India \\ ${ }^{2}$ Department of Mathematics, Central University of Haryana, Pali, India \\ ${ }^{3}$ Mathematics Discipline, PDPM-Indian Institute of Information Technology, Design and Manufacturing, \\ Jabalpur, India \\ ${ }^{4}$ Department of Mathematics, School of Advanced Sciences, Vellore Institute of Technology, Vellore, India \\ ${ }^{5}$ L. 1627 Awadh Puri Colony Beniganj, Phase-III, Opposite-Industrial Training Institute (I.T.I.), Faizabad, India \\ ${ }^{6}$ Department of Mathematics, Indira Gandhi National Tribal University, Lalpur, India \\ Email: vdrai1988@gmail.com, rdubeyjiya@gmail.com,dmrai23@gmail.com, *lakshminarayanmishra04@gmail.com, \\ vishnunarayanmishra@gmail.com
}

How to cite this paper: Vandana, Dubey, R., Deepmala, Mishra, L.N. and Mishra, V.N. (2018) Duality Relations for a Class of a Multiobjective Fractional Programming Problem Involving Support Functions. American Journal of Operations Research, 8 , 294-311.

https://doi.org/10.4236/ajor.2018.84017

Received: April 21, 2018

Accepted: June 30, 2018

Published: July 3, 2018

Copyright $(9) 2018$ by authors and Scientific Research Publishing Inc. This work is licensed under the Creative Commons Attribution International License (CC BY 4.0).

http://creativecommons.org/licenses/by/4.0/

\section{c) (i) Open Access}

\begin{abstract}
In this article, for a differentiable function $H: R^{n} \times R^{n} \rightarrow R$, we introduce the definition of the higher-order $(V, \alpha, \beta, \rho, d)$-invexity. Three duality models for a multiobjective fractional programming problem involving nondifferentiability in terms of support functions have been formulated and usual duality relations have been established under the higher-order $(V, \alpha, \beta, \rho, d)$-invex assumptions.
\end{abstract}

\section{Keywords}

Efficient Solution, Support Function, Multiobjective Fractional Programming, Generalized Invexity

\section{Introduction}

Consider the following nonlinear programming problem (P) Minimize $f(x)$ subject to $g(x) \leq 0$, where $f: R^{n} \rightarrow R$ and $g: R^{n} \rightarrow R$ are twice differentiable functions. The Mangasarian [1] second-order dualof (P) is (DP) Maximize

$$
f(u)-y^{\mathrm{T}} g(u)-\frac{1}{2} p^{\mathrm{T}} \nabla^{2}\left[f(u)-y^{\mathrm{T}} g(u)\right] p
$$

such that $\nabla\left[f(u)-y^{\mathrm{T}} g(u)\right]+\nabla^{2}\left[f(u)-y^{\mathrm{T}} g(u)\right] p=0$

*Corresponding author. 
By introducing two differentiable functions $H: R^{n} \times R^{n} \rightarrow R$ and $K: R^{n} \times R^{n} \rightarrow R^{m}$, Mangasarian [1] formulated the following higher-order dual of (P): (DP) ${ }_{1}$ Maximize

$$
f(u)-y^{\mathrm{T}} g(u)+H(u, p)-y^{\mathrm{T}} K(u, p)
$$

such that $\nabla_{p} H(u, p)-\nabla_{p}\left[y^{\mathrm{T}} K(u, p)\right]=0, y \geq 0$, where $\nabla_{p} H(u, p)$ denotes the $n \times 1$ gradient of $H(u, p)$ with respect to $p$ and $\nabla_{p}\left(y^{\mathrm{T}} K(u, p)\right)$ denotes the $n \times 1$, gradient of $y^{\mathrm{T}} K(u, p)$ with respect to $p$.

Further, Egudo [2] studied the following multiobjective fractional programming problem: (MFPP) Minimize

$$
G(x)=\left(\frac{f_{1}(x)}{g_{1}(x)}, \frac{f_{2}(x)}{g_{2}(x)}, \cdots, \frac{f_{k}(x)}{g_{k}(x)}\right)
$$

subject to

$$
x \in X^{0}=\left\{x \in X \subset R^{n}: h_{j}(x) \leq 0, j \in M\right\},
$$

where $f=\left(f_{1}, f_{2}, \cdots, f_{k}\right): X \rightarrow R^{k}, g=\left(g_{1}, g_{2}, \cdots, g_{k}\right): X \rightarrow R^{k}$ and $h=\left(h_{1}, h_{2}, \cdots, h_{m}\right): X \rightarrow R^{m}$ are differentiable on $X$. Also, he discussed duality results for Mond-Weir and Schaible type dual programs under generalized convexity.

For the nondifferentiable multiobjective programming problem: (MPP) Minimize

$$
G(x)=\left(f_{1}(x)+S\left(x \mid C_{1}\right), f_{2}(x)+S\left(x \mid C_{2}\right), \cdots, f_{k}(x)+S\left(x \mid C_{k}\right)\right)
$$

subject to $x \in X^{0}=\left\{x \in X \subset R^{n}: g_{j}(x)+S\left(x \mid E_{j}\right) \leq 0, j=1,2, \cdots, m\right\}$, where $f_{i}: X \rightarrow R(i=1,2, \cdots, k)$ and $g_{j}: X \rightarrow R(j=1,2, \cdots, m)$ are differentiable functions. $C_{i}$ and $E_{j}$ are compact convex sets in $R^{n}$ and $S\left(x \mid C_{i}\right)(i=1,2, \cdots, k)$ and $S\left(x \mid E_{j}\right)(j=1,2, \cdots, m)$ denote the support functions of compact convex sets, various researchers have worked. Gulati and Agarwal [3] introduced the higher-order Wolfe-type dual model of (MPP) and proved duality theorems under higher-order $(F, \rho, \rho, d)$-type $I$-assumptions.

In last several years, various optimality and duality results have been obtained for multiobjective fractional programming problems. In Chen [4], multiobjective fractional problem and its duality theorems have been considered under higherorder $(F, \alpha, \rho, d)$-convexity. Later on, Suneja et al. [5] discussed higher-order Mond-Weir and Schaible type nondifferentiable dual programs and their duality theorems under higher-order $(F, \rho, \sigma)$-type $I$-assumptions. Several researchers have also worked in this directions such as ([6] [7]).

In this paper, we first introduce the definition of higher-order $(V, \alpha, \beta, \rho, d)$ invex with respect to differentiable function $H: R^{n} \times R^{n} \rightarrow R$. We also construct a nontrivial numerical example which illustrates the existence of such a function. We then formulate three higher-order dual problems corresponding to the multiobjective nondifferentiable fractional programming problem. Further, we 
establish usual duality relations for these primal-dual pairs under aforesaid assumptions.

\section{Preliminaries}

Let $X \subseteq R^{n}$ be an open set and $\phi: X \rightarrow R, H: X \times R^{n} \rightarrow R$ be differentiable functions. $\alpha, \beta: X \times X \rightarrow R_{+} \backslash\{0\}, \eta: X \times X \rightarrow R^{n}, \rho \in R^{n}$ and $\theta: X \times X \rightarrow R^{n}$

Definition 2.1. $\phi$ is said to be (strictly) higher-order $(V, \alpha, \beta, \rho, \theta)$-invex at $u$ with respect to $H(u, p)$, if there exist $\eta, \alpha, \beta, \rho$ and $\theta$ such that, for any $x \in X$ and $p \in R^{n}$,

$$
\begin{aligned}
& \alpha(x, u)[\phi(x)-\phi(u)](>) \geq \eta^{\mathrm{T}}(x, u)\left(\nabla \phi(u)+\nabla_{p} H(u, p)\right) \\
& +\beta(x, u)\left[H(u, p)-p^{\mathrm{T}} \nabla_{p} H(u, p)\right]+\rho\|\theta(x, u)\|^{2} .
\end{aligned}
$$

Example 2.1. Let $\phi: R \rightarrow R$ be such that $\phi(x)=x^{4}+x^{2}+1$.

Let

$$
\eta(x, u)=\frac{1}{2}\left(x^{2}+u^{2}\right), H(u, p)=-2 p(x+1)^{2} .
$$

Also, suppose

$$
\alpha(x, u)=1, \beta(x, u)=2, \rho=-1,\|\theta(x, u)\|=\left(x^{2}+u^{2}\right)^{\frac{1}{2}} .
$$

Now,

$$
\begin{gathered}
\xi=\alpha(x, u)[\phi(x)-\phi(u)]-\eta^{\mathrm{T}}(x, u)\left(\nabla \phi(u)+\nabla_{p} H(u, p)\right) \\
-\beta(x, u)\left[H(u, p)-p^{\mathrm{T}} \nabla_{p} H(u, p)\right]-\rho\|\theta(x, u)\|^{2} . \\
\xi=\left(x^{4}+x^{2}-u^{4}-u^{2}\right)-\frac{1}{2}\left(x^{2}+u^{2}\right)\left[4 u^{3}+2 u-2(u+1)^{2}\right]-\left(x^{2}+u^{2}\right) \\
\left.\xi=x^{4}+x^{2} \quad \text { at } u=0\right) . \\
\geq 0, \forall x \in R .
\end{gathered}
$$

Hence, $\phi$ is higher-order $(V, \alpha, \beta, \rho, \theta)$-invex at $u=0$ with respect to $H(u, p)$.

Remark 2.1.

1) If $H(u, p)=0$, then the Definition 2.1 reduces to $(V, \rho)$-invex function introduced by Kuk et al. [8].

2) If $H(u, p)=0$ and $\rho=0$, then the Definition 2.1 becomes that of $V$-invexity introduced by Jeyakumar and Mond [9].

3) If $H(u, p)=\frac{1}{2} p^{\mathrm{T}} \nabla^{2} \phi(u) p, \alpha(x, u)=0$ and $\rho=0$, then above definition yields in $\eta$-bonvexity given by Pandey [10].

4) If $\beta=1$, then the Definition 2.1 reduced in $(V, \alpha, \rho, \theta)$-invex given by Gulati and Geeta [11].

A differentiable function $f=\left(f_{1}, f_{2}, \cdots, f_{k}\right): X \rightarrow R^{k}$ is $(V, \alpha, \beta, \rho, \theta)$-invex if for all $i=1,2, \cdots, k, f_{i}$ is $\left(V, \alpha_{i}, \beta_{i}, \rho_{i}, \theta_{i}\right)$-invex.

Definition 2.2. [12]. Let $C$ be a compact convex set in $R^{n}$. The support 
function of $C$ is defined by

$$
S(x \mid C)=\max \left\{x^{\mathrm{T}} y: y \in C\right\} .
$$

\section{Problem Formulation}

Consider the multiobjective programming problem with support function given as: (MFP) Minimize

$$
F(x)=\left\{\frac{f_{1}(x)+S\left(x \mid C_{1}\right)}{g_{1}(x)-S\left(x \mid D_{1}\right)}, \frac{f_{2}(x)+S\left(x \mid C_{2}\right)}{g_{2}(x)-S\left(x \mid D_{2}\right)}, \cdots, \frac{f_{k}(x)+S\left(x \mid C_{k}\right)}{g_{k}(x)-S\left(x \mid D_{k}\right)}\right\}
$$

subject to $x \in X^{0}=\left\{x \in X \subset R^{n}: h_{j}(x)+S\left(x \mid E_{j}\right) \leq 0, j=1,2, \cdots, m\right\}$, where $f=\left(f_{1}, f_{2}, \cdots, f_{k}\right): X \rightarrow R^{k}, g=\left(g_{1}, g_{2}, \cdots, g_{k}\right): X \rightarrow R^{k}$ and $h=\left(h_{1}, h_{2}, \cdots, h_{m}\right): X \rightarrow R^{m}$ are differentiable on $X, f_{i}()+.S\left(. \mid C_{i}\right) \geq 0$ and $g_{i}()-.S\left(. \mid D_{i}\right)>0$. Let $H_{i}: X \times R^{n} \rightarrow R$ be differentiable functions, $C_{i}, D_{i}$ and $E_{j}$ are compact convex sets in $R^{n}$, for all $i=1,2, \cdots, k, j=1,2, \cdots, m$.

Definition 3.1. [3]. A point $x^{0} \in X^{0}$ is said to be an efficient solution (or Pareto optimal) of (MFP), if there exists no $x \in X^{0}$ such that for every

$$
i=1,2, \cdots, k, \frac{f_{i}(x)+S\left(x \mid C_{i}\right)}{g_{i}(x)-S\left(x \mid D_{i}\right)} \leq \frac{f_{i}\left(x^{0}\right)+S\left(x^{0} \mid C_{i}\right)}{g_{i}\left(x^{0}\right)-S\left(x^{0} \mid D_{i}\right)}
$$

and for some $r=1,2, \cdots, k$,

$$
\frac{f_{r}(x)+S\left(x \mid C_{r}\right)}{g_{r}(x)-S\left(x \mid D_{r}\right)}<\frac{f_{r}\left(x^{0}\right)+S\left(x^{0} \mid C_{r}\right)}{g_{r}\left(x^{0}\right)-S\left(x^{0} \mid D_{r}\right)} .
$$

We now state theorems 3.1-3.2, whose proof follows on the lines [13].

Theorem 3.1. For some $t$, if $f_{t}()+.(.)^{\mathrm{T}} z_{t}$ and $-\left(g_{t}()-.(.)^{\mathrm{T}} v_{t}\right)$ are higherorder $\left(V, \alpha_{t}, \beta_{t}, \rho_{t}, \theta_{t}\right)$-invex at $u$ with respect to $H_{t}(u, p)$ for same $\eta(x, u)$. Then, the fractional function $\left(\frac{f_{t}(.)+(.)^{\mathrm{T}} z_{t}}{g_{t}(.)-(.)^{\mathrm{T}} v_{t}}\right)$ is higher-order $\left(V, \bar{\alpha}_{t}, \bar{\beta}_{t}, \bar{\rho}_{t}, \bar{\theta}_{t}\right)$ -invex at $u$ with respect to $\bar{H}_{t}(u, p)$, where

$$
\begin{gathered}
\bar{\alpha}_{t}(x, u)=\left(\frac{g_{t}(x)-x^{\mathrm{T}} v_{t}}{g_{t}(u)-u^{\mathrm{T}} v_{t}}\right) \alpha_{t}(x, u), \quad \bar{\beta}_{t}(x, u)=\beta_{t}(x, u), \\
\bar{\theta}_{t}(x, u)=\theta_{t}(x, u)\left(\frac{1}{g_{t}(u)-u^{\mathrm{T}} v_{t}}+\frac{f_{t}(u)+u^{\mathrm{T}} z_{t}}{\left(g_{t}(u)-u^{\mathrm{T}} v_{t}\right)^{2}}\right)^{\frac{1}{2}}, \bar{\rho}_{t}(x, u)=\rho_{t}(x, u)
\end{gathered}
$$

and

$$
\bar{H}_{t}(u, p)=\left(\frac{1}{g_{t}(u)-u^{\mathrm{T}} v_{t}}+\frac{f_{t}(u)+u^{\mathrm{T}} z_{t}}{\left(g_{t}(u)-u^{\mathrm{T}} v_{t}\right)^{2}}\right) H_{t}(u, p) .
$$

Theorem 3.2. In Theorem 3.1 ,if either $-\left(g_{t}()-.(.)^{\mathrm{T}} v_{t}\right)$ is strictly higher- 
order $\left(V, \alpha_{t}, \beta_{t}, \rho_{t}, \theta_{t}\right)$-invex at $u$ with respect to $H_{t}(u, p)$ and $\left(f_{t}()-.(.)^{\mathrm{T}} z_{t}\right)>0$ or $\left(f_{t}()-.(.)^{\mathrm{T}} z_{t}\right)$ is strictly higher-order $\left(V, \alpha_{t}, \beta_{t}, \rho_{t}, \theta_{t}\right)$ - invex at $u$ with respect to $H_{t}(u, p)$, then $\left(\frac{f_{t}(.)+(.)^{\mathrm{T}} z_{t}}{g_{t}(.)-(.)^{\mathrm{T}} z_{t}}\right)$ is strictly higherorder $\left(V, \bar{\alpha}_{t}, \bar{\beta}_{t}, \bar{\rho}_{t}, \bar{\theta}_{t}\right)$-invex at $u \in X$ with respect to $\bar{H}_{t}(u, p)$.

Theorem 3.3 (Necessary Condition) [14]. Assume that $\bar{x}$ is an efficient solution of (MFP) and the Slater's constraint qualification is satisfied on $X$. Then there exist $\bar{\lambda}_{i}>0, \bar{\mu}_{j} \in R^{m}, \bar{z}_{i} \in R^{n}, \bar{v}_{i} \in R^{n}$ and $\bar{w}_{j} \in R^{m}, i=1,2, \cdots, k, j=1,2, \cdots, m$, such that

$$
\begin{gathered}
\sum_{i=1}^{k} \bar{\lambda}_{i} \nabla\left(\frac{f_{i}(\bar{x})+\bar{x}^{\mathrm{T}} \bar{z}_{i}}{g_{i}(\bar{x})-\bar{x}^{\mathrm{T}} \bar{v}_{i}}\right)+\sum_{j=1}^{m} \bar{\mu}_{j} \nabla\left(h_{j}(\bar{x})+\bar{x}^{\mathrm{T}} \bar{w}_{j}\right)=0, \\
\sum_{j=1}^{m} \bar{\mu}_{j}\left(h_{j}(\bar{x})+\bar{x}^{\mathrm{T}} \bar{w}_{j}\right)=0, \\
\bar{x}^{\mathrm{T}} \bar{z}_{i}=S\left(\bar{x} \mid C_{i}\right), \bar{z}_{i} \in C_{i}, i=1,2, \cdots, k, \\
\bar{x}^{\mathrm{T}} \bar{v}_{i}=S\left(\bar{x} \mid D_{i}\right), \bar{v}_{i} \in D_{i}, i=1,2, \cdots, k, \\
\bar{x}^{\mathrm{T}} \bar{w}_{j}=S\left(\bar{x} \mid E_{j}\right), \bar{w}_{j} \in E_{j}, j=1,2, \cdots, m, \\
\bar{\lambda}_{i}>0, i=1,2, \cdots, k, \bar{\mu}_{j} \geq 0, j=1,2, \cdots, m .
\end{gathered}
$$

Theorem 3.4. (Sufficient Condition). Let $u$ be a feasible solution of (MFP). Then, there exist $\lambda_{i}>0, i=1,2, \cdots, k$ and $\mu_{j} \geq 0, j=1,2, \cdots, m$, such that

$$
\begin{gathered}
\sum_{i=1}^{k} \lambda_{i} \nabla\left(\frac{f_{i}(u)+u^{\mathrm{T}} z_{i}}{g_{i}(u)-u^{\mathrm{T}} v_{i}}\right)+\sum_{j=1}^{m} \mu_{j} \nabla\left(h_{j}(u)+u^{\mathrm{T}} w_{j}\right)=0, \\
\sum_{j=1}^{m} \mu_{j}\left(h_{j}(u)+u^{\mathrm{T}} w_{j}\right)=0, \\
u^{\mathrm{T}} z_{i}=S\left(u \mid C_{i}\right), z_{i} \in C_{i}, i=1,2, \cdots, k, \\
u^{\mathrm{T}} v_{i}=S\left(u \mid D_{i}\right), v_{i} \in D_{i}, i=1,2, \cdots, k, \\
u^{\mathrm{T}} w_{j}=S\left(u \mid E_{j}\right), w_{j} \in E_{j}, j=1,2, \cdots, m, \\
\bar{\lambda}_{i}>0, i=1,2, \cdots, k, \bar{\mu}_{j} \geq 0, j=1,2, \cdots, m .
\end{gathered}
$$

Let, for $i=1,2, \cdots, k, j=1,2, \cdots, m$,

1) $\left(f_{i}()+.(.)^{\mathrm{T}} z_{i}\right)$ and $-\left(g_{i}()-.(.)^{\mathrm{T}} v_{i}\right)$ be higher-order $\left(V, \alpha_{i}^{1}, \beta_{i}^{1}, \rho_{i}^{1}, \theta_{i}^{1}\right)$ invex at $u$ with respect to $H_{i}(u, p)$,

2) $\left(h_{j}()+.(.)^{\mathrm{T}} w_{j}\right)$ be higher-order $\left(V, \alpha_{j}^{2}, \beta_{j}^{2}, \rho_{j}^{2}, \theta_{j}^{2}\right)$-invex at $u$ with respect to $G_{j}(u, p)$,
3) $\sum_{i=1}^{k} \lambda_{i} \bar{\rho}_{i}^{1}\left\|\bar{\theta}_{i}^{1}(x, u)\right\|^{2}+\sum_{j=1}^{m} \mu_{j} \rho_{j}^{2}\left\|\theta_{j}^{2}(x, u)\right\|^{2} \geq 0$,
4) $\sum_{i=1}^{k} \lambda_{i}\left(\nabla_{p} \bar{H}_{i}(u, p)\right)+\sum_{j=1}^{m} \mu_{j}\left(\nabla_{p} G_{j}(u, p)\right)=0$, 
$\sum_{i=1}^{k} \lambda_{i}\left(\bar{H}_{i}(u, p)-p^{\mathrm{T}} \nabla_{p} \bar{H}_{i}(u, p)\right) \geq 0$ and $\sum_{j=1}^{m} \mu_{j}\left(G_{j}(u, p)-p^{\mathrm{T}} \nabla_{p} G_{j}(u, p)\right) \geq 0$,

5) $\alpha_{i}^{1}(x, u)=\alpha_{j}^{2}(x, u)=\beta_{i}^{1}(x, u)=\beta_{j}^{2}(x, u)=\alpha(x, u)$,

where

$$
\begin{gathered}
\bar{\alpha}_{i}(x, u)=\left(\frac{g_{i}(x)-x^{\mathrm{T}} v_{i}}{g_{i}(u)-u^{\mathrm{T}} v_{i}}\right) \alpha_{i}(x, u), \quad \bar{\beta}_{i}(x, u)=\beta_{i}(x, u), \\
\bar{\theta}_{i}(x, u)=\theta_{i}(x, u)\left(\frac{1}{g_{i}(u)-u^{\mathrm{T}} v_{i}}+\frac{f_{i}(u)+u^{\mathrm{T}} z_{i}}{\left(g_{i}(u)-u^{\mathrm{T}} v_{i}\right)^{2}}\right)^{\frac{1}{2}}
\end{gathered}
$$

and $\bar{\rho}_{i}(x, u)=\rho_{i}(x, u)$.

Then, $u$ is an efficient solution of (MFP).

Proof. Suppose $u$ is not an efficient solution of (MFP). Then there exists $x \in X^{0}$ such that

$$
\frac{f_{i}(x)+S\left(x \mid C_{i}\right)}{g_{i}(x)-S\left(x \mid D_{i}\right)} \leq \frac{f_{i}(u)+S\left(u \mid C_{i}\right)}{g_{i}(u)-S\left(u \mid D_{i}\right)}, \text { for all } i=1,2, \cdots, k
$$

and

$$
\frac{f_{r}(x)+S\left(x \mid C_{r}\right)}{g_{r}(x)-S\left(x \mid D_{r}\right)}<\frac{f_{r}(u)+S\left(u \mid C_{r}\right)}{g_{r}(u)-S\left(u \mid D_{r}\right)}, \text { for some } r=1,2, \cdots, k,
$$

which implies

$$
\begin{aligned}
\frac{f_{i}(x)+x^{\mathrm{T}} z_{i}}{g_{i}(x)-x^{\mathrm{T}} v_{i}} & \leq \frac{f_{i}(x)+S\left(x \mid C_{i}\right)}{g_{i}(x)-S\left(x \mid D_{i}\right)} \leq \frac{f_{i}(u)+S\left(u \mid C_{i}\right)}{g_{i}(u)-S\left(u \mid D_{i}\right)} \\
& =\frac{f_{i}(u)+u^{\mathrm{T}} z_{i}}{g_{i}(u)-u^{\mathrm{T}} v_{i}}, \text { for all } i=1,2, \cdots, k
\end{aligned}
$$

and

$$
\begin{aligned}
\frac{f_{r}(x)+x^{\mathrm{T}} z_{r}}{g_{r}(x)-x^{\mathrm{T}} v_{r}} & \leq \frac{f_{r}(x)+S\left(x \mid C_{r}\right)}{g_{r}(x)-S\left(x \mid D_{r}\right)}<\frac{f_{r}(u)+S\left(u \mid C_{r}\right)}{g_{r}(u)-S\left(u \mid D_{r}\right)} \\
& =\frac{f_{r}(u)+u^{\mathrm{T}} z_{r}}{g_{r}(u)-u^{\mathrm{T}} v_{r}}, \text { for some } r=1,2, \cdots, k .
\end{aligned}
$$

Since $\lambda_{i}>0, i=1,2, \cdots, k$, inequalities (13) and (14) gives

$$
\sum_{i=1}^{k} \lambda_{i}\left(\frac{f_{i}(x)+x^{\mathrm{T}} z_{i}}{g_{i}(x)-x^{\mathrm{T}} v_{i}}-\frac{f_{i}(u)+u^{\mathrm{T}} z_{i}}{g_{i}(u)-u^{\mathrm{T}} v_{i}}\right)<0 .
$$

From Theorem 3.1, for each $i, 1 \leq i \leq k,\left(\frac{f_{i}(.)+(.)^{\mathrm{T}} z_{i}}{g_{i}(.)-(.)^{\mathrm{T}} v_{i}}\right)$

is higher-order $\left(V, \bar{\alpha}_{i}^{1}, \bar{\beta}_{i}^{1}, \bar{\rho}_{i}^{1}, \bar{\theta}_{i}^{1}\right)$-invex at $u \in X^{0}$ with respect to $\bar{H}_{i}(u, p)$, we have

$$
\bar{\alpha}_{i}^{1}(x, u)\left[\frac{f_{i}(x)+x^{\mathrm{T}} z_{i}}{g_{i}(x)-x^{\mathrm{T}} v_{i}}-\frac{f_{i}(u)+u^{\mathrm{T}} z_{i}}{g_{i}(u)-u^{\mathrm{T}} v_{i}}\right]
$$




$$
\begin{aligned}
& \geq \eta^{\mathrm{T}}(x, u)\left[\nabla\left(\frac{f_{i}(u)+u^{\mathrm{T}} z_{i}}{g_{i}(u)-u^{\mathrm{T}} v_{i}}\right)+\nabla_{p} \bar{H}_{i}(u, p)\right] \\
& \quad+\bar{\beta}_{i}^{1}(x, u)\left[\bar{H}_{i}(u, p)-p^{\mathrm{T}} \nabla_{p} \bar{H}_{i}(u, p)\right]+\bar{\rho}_{i}^{1}\left\|\bar{\theta}_{i}^{1}(x, u)\right\|^{2} .
\end{aligned}
$$

where

$$
\begin{gathered}
\bar{\alpha}_{i}(x, u)=\left(\frac{g_{i}(x)-x^{\mathrm{T}} v_{i}}{g_{i}(u)-u^{\mathrm{T}} v_{i}}\right) \alpha_{i}(x, u), \bar{\beta}_{i}(x, u)=\beta_{i}(x, u), \\
\bar{\theta}_{i}(x, u)=\theta_{i}(x, u)\left(\frac{1}{g_{i}(u)-u^{\mathrm{T}} v_{i}}+\frac{f_{i}(u)+u^{\mathrm{T}} z_{i}}{\left(g_{i}(u)-u^{\mathrm{T}} v_{i}\right)^{2}}\right)^{\frac{1}{2}}, \bar{\rho}_{i}(x, u)=\rho_{i}(x, u) \\
\text { and } \bar{H}_{i}(u, p)=\left(\frac{1}{g_{i}(u)-u^{\mathrm{T}} v_{i}}+\frac{f_{i}(u)+u^{\mathrm{T}} z_{i}}{\left(g_{i}(u)-u^{\mathrm{T}} v_{i}\right)^{2}}\right) H_{i}(u, p) .
\end{gathered}
$$

By hypothesis 2), we get

$$
\begin{aligned}
& \alpha_{j}^{2}(x, u)\left[h_{j}(x)+x^{\mathrm{T}} w_{j}-\left(h_{j}(u)+u^{\mathrm{T}} w_{j}\right)\right] \\
& \geq \eta^{\mathrm{T}}(x, u)\left[\nabla\left(h_{j}(u)+u^{\mathrm{T}} w_{j}\right)+\nabla_{p} G_{j}(u, p)\right] \\
& \quad+\beta_{j}^{2}(x, u)\left[G_{j}(u, p)-p^{\mathrm{T}} \nabla_{p} G_{j}(u, p)\right]+\rho_{j}^{2}\left\|\theta_{j}^{2}(x, u)\right\|^{2} .
\end{aligned}
$$

Adding the two inequalities after multiplying (16) by $\lambda_{i}$ and (17) by $\mu_{j}$, we obtain

$$
\begin{aligned}
& \sum_{i=1}^{k} \lambda_{i} \bar{\alpha}_{i}^{1}(x, u)\left[\frac{f_{i}(x)+x^{\mathrm{T}} z_{i}}{g_{i}(x)-x^{\mathrm{T}} v_{i}}-\frac{f_{i}(u)+u^{\mathrm{T}} z_{i}}{g_{i}(u)-u^{\mathrm{T}} v_{i}}\right] \\
& +\sum_{j=1}^{m} \mu_{j} \alpha_{j}^{2}(x, u)\left[h_{j}(x)+x^{\mathrm{T}} w_{j}-\left(h_{j}(u)+u^{\mathrm{T}} w_{j}\right)\right] \\
& \geq \eta^{\mathrm{T}}(x, u) \sum_{i=1}^{k} \lambda_{i}\left[\nabla\left(\frac{f_{i}(u)+u^{\mathrm{T}} z_{i}}{g_{i}(u)-u^{\mathrm{T}} v_{i}}\right)+\nabla_{p} \bar{H}_{i}(u, p)\right] \\
& +\eta^{\mathrm{T}}(x, u) \sum_{j=1}^{m} \mu_{j}\left[\nabla\left(h_{j}(u)+u^{\mathrm{T}} w_{j}\right)+\nabla_{p} G_{j}(u, p)\right] \\
& +\sum_{i=1}^{k} \lambda_{i} \bar{\beta}_{i}(x, u)\left[\bar{H}_{i}(u, p)-p^{\mathrm{T}} \nabla_{p} \bar{H}_{i}(u, p)\right] \\
& +\sum_{j=1}^{m} \mu_{j} \beta_{j}^{2}(x, u)\left[G_{j}(u, p)-p^{\mathrm{T}} \nabla_{p} G_{j}(u, p)\right] \\
& +\sum_{i=1}^{k} \lambda_{i} \bar{\rho}_{i}^{1}\left\|\bar{\theta}_{i}^{1}(x, u)\right\|^{2}+\sum_{j=1}^{m} \mu_{j} \rho_{j}^{2}\left\|\theta_{j}^{2}(x, u)\right\|^{2} .
\end{aligned}
$$

Using hypothesis 3)-4), we get

$$
\begin{aligned}
& \sum_{i=1}^{k} \lambda_{i}\left[\frac{f_{i}(x)+x^{\mathrm{T}} z_{i}}{g_{i}(x)-x^{\mathrm{T}} v_{i}}-\frac{f_{i}(u)+u^{\mathrm{T}} z_{i}}{g_{i}(u)-u^{\mathrm{T}} v_{i}}\right]+\sum_{j=1}^{m} \mu_{j}\left[h_{j}(x)+x^{\mathrm{T}} w_{j}-\left(h_{j}(u)+u^{\mathrm{T}} w_{j}\right)\right] \\
& \geq \eta^{\mathrm{T}}(x, u) \sum_{i=1}^{k} \lambda_{i} \nabla\left(\frac{f_{i}(u)+u^{\mathrm{T}} z_{i}}{g_{i}(u)-u^{\mathrm{T}} v_{i}}\right)+\eta^{\mathrm{T}}(x, u) \sum_{j=1}^{m} \mu_{j} \nabla\left(h_{j}(u)+u^{\mathrm{T}} w_{j}\right) .
\end{aligned}
$$


Further, using (7)-(8), therefore

$$
\sum_{i=1}^{k} \lambda_{i}\left[\frac{f_{i}(x)+x^{\mathrm{T}} z_{i}}{g_{i}(x)-x^{\mathrm{T}} v_{i}}-\frac{f_{i}(u)+u^{\mathrm{T}} z_{i}}{g_{i}(u)-u^{\mathrm{T}} v_{i}}\right]+\sum_{j=1}^{m} \mu_{j}\left[h_{j}(x)+x^{\mathrm{T}} w_{j}\right] \geq 0 .
$$

Since $x$ is feasible solution for (MFP), it follows that

$$
\sum_{i=1}^{k} \lambda_{i}\left(\frac{f_{i}(x)+x^{\mathrm{T}} z_{i}}{g_{i}(x)-x^{\mathrm{T}} v_{i}}\right) \geq \sum_{i=1}^{k} \lambda_{i}\left(\frac{f_{i}(u)+u^{\mathrm{T}} z_{i}}{g_{i}(u)-u^{\mathrm{T}} v_{i}}\right) .
$$

This contradicts (15). Therefore, $u$ is an efficient solution of (MFP).

\section{Duality Model-I}

Consider the following dual (MFD), of (MFP): (MFD) ${ }_{1}$ Maximize

$$
\begin{aligned}
& {\left[\frac{f_{1}(u)+u^{\mathrm{T}} z_{1}}{g_{1}(u)-u^{\mathrm{T}} v_{1}}+\sum_{j=1}^{m} \mu_{j}\left(h_{j}(u)+u^{\mathrm{T}} w_{j}\right)+\left(\bar{H}_{1}(u, p)-p^{\mathrm{T}} \nabla_{p} \bar{H}_{1}(u, p)\right)\right.} \\
& +\sum_{j=1}^{m} \mu_{j}\left(G_{j}(u, p)-p^{\mathrm{T}} \nabla_{p} G_{j}(u, p)\right), \cdots, \\
& \frac{f_{k}(u)+u^{\mathrm{T}} z_{k}}{g_{k}(u)-u^{\mathrm{T}} v_{k}}+\sum_{j=1}^{m} \mu_{j}\left(h_{j}(u)+u^{\mathrm{T}} w_{j}\right)+\left(\bar{H}_{k}(u, p)-p^{\mathrm{T}} \nabla_{p} \bar{H}_{k}(u, p)\right) \\
& \left.+\sum_{j=1}^{m} \mu_{j}\left(G_{j}(u, p)-p^{\mathrm{T}} \nabla_{p} G_{j}(u, p)\right)\right]
\end{aligned}
$$

subject to

$$
\begin{gathered}
\sum_{i=1}^{k} \lambda_{i} \nabla\left(\frac{f_{i}(u)+u^{\mathrm{T}} z_{i}}{g_{i}(u)-u^{\mathrm{T}} v_{i}}\right)+\sum_{j=1}^{m} \mu_{j} \nabla\left(h_{j}(u)+u^{\mathrm{T}} w_{j}\right) \\
+\sum_{i=1}^{k} \lambda_{i} \nabla_{p} \bar{H}_{i}(u, p)+\sum_{j=1}^{m} \mu_{j} \nabla_{p} G_{j}(u, p)=0, \\
z_{i} \in C_{i}, v_{i} \in D_{i}, w_{j} \in E_{j}, i=1,2, \cdots, k, j=1,2, \cdots, m, \\
\mu_{j} \geq 0, \lambda_{i}>0, \sum_{i=1}^{k} \lambda_{i}=1, i=1,2, \cdots, k, j=1,2, \cdots, m .
\end{gathered}
$$

Let $Z^{0}$ be feasible solution for (MFD) .

Theorem 4.1. (Weak duality theorem). Let $x \in X^{0}$ and $(u, z, v, \mu, \lambda, w, p) \in Z^{0}$. Suppose that

1) for any $i=1,2, \cdots, k,\left(f_{i}()+.(.)^{\mathrm{T}} z_{i}\right)$ and $-\left(g_{i}()-.(.)^{\mathrm{T}} v_{i}\right)$ are higherorder $\left(V, \alpha_{i}^{1}, \beta_{i}^{1}, \rho_{i}^{1}, \theta_{i}^{1}\right)$-invex at $u$ with respect to $H_{i}(u, p)$,

2) for any $j=1,2, \cdots, m,\left(h_{j}()+.(.)^{\mathrm{T}} w_{j}\right)$ is higher-order $\left(V, \alpha_{j}^{2}, \beta_{j}^{2}, \rho_{j}^{2}, \theta_{j}^{2}\right)$ -invex at $u$ with respect to $G_{j}(u, p)$,

3) $\sum_{i=1}^{k} \lambda_{i} \bar{\rho}_{i}^{1}\left\|\bar{\theta}_{i}^{1}(x, u)\right\|^{2}+\sum_{j=1}^{m} \mu_{j} \rho_{j}^{2}\left\|\theta_{j}^{2}(x, u)\right\|^{2} \geq 0$.

4) $\bar{\alpha}_{i}^{1}(x, u)=\alpha_{j}^{2}(x, u)=\beta_{i}^{1}(x, u)=\beta_{j}^{2}(x, u)=\alpha(x, u), \forall i=1,2, \cdots, k$, $j=1,2, \cdots, m$,

where $\bar{\alpha}_{t}(x, u)=\left(\frac{g_{t}(x)-x^{\mathrm{T}} v_{t}}{g_{t}(u)-u^{\mathrm{T}} v_{t}}\right) \alpha_{t}(x, u), \quad \bar{\beta}_{t}(x, u)=\beta_{t}(x, u)$, 


$$
\begin{aligned}
& \bar{\theta}_{t}(x, u)=\theta_{t}(x, u)\left(\frac{1}{g_{t}(u)-u^{\mathrm{T}} v_{t}}+\frac{f_{t}(u)+u^{\mathrm{T}} z_{t}}{\left(g_{t}(u)-u^{\mathrm{T}} v_{t}\right)^{2}}\right)^{\frac{1}{2}}, \bar{\rho}_{t}(x, u)=\rho_{t}(x, u) \text { and } \\
& \bar{H}_{t}(u, p)=\left(\frac{1}{g_{t}(u)-u^{\mathrm{T}} v_{t}}+\frac{f_{t}(u)+u^{\mathrm{T}} z_{t}}{\left(g_{t}(u)-u^{\mathrm{T}} v_{t}\right)^{2}}\right) H_{t}(u, p) .
\end{aligned}
$$

Then, the following cannot hold

$$
\begin{aligned}
& \frac{f_{i}(x)+S\left(x \mid C_{i}\right)}{g_{i}(x)-S\left(x \mid D_{i}\right)} \\
& \leq \frac{f_{i}(u)+u^{\mathrm{T}} z_{i}}{g_{i}(u)-u^{\mathrm{T}} v_{i}}+\sum_{j=1}^{m} \mu_{j}\left(h_{j}(u)+u^{\mathrm{T}} w_{j}\right)+\left(\bar{H}_{i}(u, p)-p^{\mathrm{T}} \nabla_{p} \bar{H}_{i}(u, p)\right) \\
& +\sum_{j=1}^{m} \mu_{j}\left(G_{j}(u, p)-p^{\mathrm{T}} \nabla_{p} G_{j}(u, p)\right), \text { for all } i=1,2, \cdots, k
\end{aligned}
$$

and

$$
\begin{aligned}
& \frac{f_{r}(x)+S\left(x \mid C_{r}\right)}{g_{r}(x)-S\left(x \mid D_{r}\right)} \\
& <\frac{f_{r}(u)+u^{\mathrm{T}} z_{r}}{g_{r}(u)-u^{\mathrm{T}} v_{r}}+\sum_{j=1}^{m} \mu_{j}\left(h_{j}(u)+u^{\mathrm{T}} w_{j}\right)+\left(\bar{H}_{r}(u, p)-p^{\mathrm{T}} \nabla_{p} \bar{H}_{r}(u, p)\right) \\
& \quad+\sum_{j=1}^{m} \mu_{j}\left(G_{j}(u, p)-p^{\mathrm{T}} \nabla_{p} G_{j}(u, p)\right), \text { for some } r=1,2, \cdots, k .
\end{aligned}
$$

Proof. Suppose that (22) and (23) hold, then using $\lambda_{i}>0, \sum_{i=1}^{k} \lambda_{i}=1$, $x^{\mathrm{T}} z_{i} \leq S\left(x \mid C_{i}\right), \quad x^{\mathrm{T}} v_{i} \leq S\left(x \mid D_{i}\right), \quad i=1,2, \cdots, k$, we have

$$
\begin{aligned}
\sum_{i=1}^{k} \lambda_{i}\left(\frac{f_{i}(x)+x^{\mathrm{T}} z_{i}}{g_{i}(x)-x^{\mathrm{T}} v_{i}}\right)< & \sum_{i=1}^{k} \lambda_{i}\left(\frac{f_{i}(u)+u^{\mathrm{T}} z_{i}}{g_{i}(u)-u^{\mathrm{T}} v_{i}}\right)+\sum_{j=1}^{m} \mu_{j}\left(h_{j}(u)+u^{\mathrm{T}} w_{j}\right) \\
& +\sum_{i=1}^{k} \lambda_{i}\left(\bar{H}_{i}(u, p)-p^{\mathrm{T}} \nabla_{p} \bar{H}_{i}(u, p)\right) \\
& +\sum_{j=1}^{m} \mu_{j}\left(G_{j}(u, p)-p^{\mathrm{T}} \nabla_{p} G_{j}(u, p)\right) .
\end{aligned}
$$

From hypothesis 1) and Theorem 3.1, for $i=1,2, \cdots, k,\left(\frac{f_{i}(.)+(.)^{\mathrm{T}} z_{i}}{g_{i}(.)-(.)^{\mathrm{T}} v_{i}}\right)$ is higher-order $\left(V, \bar{\alpha}_{i}^{1}, \bar{\beta}_{i}^{1}, \bar{\rho}_{i}^{1}, \bar{\theta}_{i}^{1}\right)$-invex at $u$ with respect to $\bar{H}_{i}(u, p)$, we get

$$
\begin{aligned}
& \bar{\alpha}_{i}^{1}(x, u)\left[\frac{f_{i}(x)+x^{\mathrm{T}} z_{i}}{g_{i}(x)-x^{\mathrm{T}} v_{i}}-\frac{f_{i}(u)+u^{\mathrm{T}} z_{i}}{g_{i}(u)-u^{\mathrm{T}} v_{i}}\right] \\
& \geq \eta^{\mathrm{T}}(x, u)\left[\nabla\left(\frac{f_{i}(u)+u^{\mathrm{T}} z_{i}}{g_{i}(u)-u^{\mathrm{T}} v_{i}}\right)+\nabla_{p} \bar{H}_{i}(u, p)\right] \\
& +\bar{\beta}_{i}^{1}(x, u)\left[\bar{H}_{i}(u, p)-p^{\mathrm{T}} \nabla_{p} \bar{H}_{i}(u, p)\right]+\bar{\rho}_{i}^{1}\left\|\bar{\theta}_{i}^{1}(x, u)\right\|^{2} .
\end{aligned}
$$

For any $j=1,2, \cdots, m,\left(h_{j}()+.(.)^{\mathrm{T}} w_{j}\right)$ is higher-order $\left(V, \alpha_{j}^{2}, \beta_{j}^{2}, \rho_{j}^{2}, \theta_{j}^{2}\right)$ -invex at $u$ with respect to $G_{j}(u, p)$, we have 


$$
\begin{aligned}
& \alpha_{j}^{2}(x, u)\left[h_{j}(x)+x^{\mathrm{T}} w_{j}-\left(h_{j}(u)+u^{\mathrm{T}} w_{j}\right)\right] \\
& \geq \eta^{\mathrm{T}}(x, u)\left[\nabla\left(h_{j}(u)+u^{\mathrm{T}} w_{j}\right)+\nabla_{p} G_{j}(u, p)\right] \\
& +\beta_{j}^{2}(x, u)\left[G_{j}(u, p)-p^{\mathrm{T}} \nabla_{p} G_{j}(u, p)\right]+\rho_{j}^{2}\left\|\theta_{j}^{2}(x, u)\right\|^{2} .
\end{aligned}
$$

Adding the two inequalities after multiplying (25) by $\lambda_{i}$ and (26) by $\mu_{j}$, we obtain

$$
\begin{aligned}
& \sum_{i=1}^{k} \lambda_{i} \bar{\alpha}_{i}^{1}(x, u)\left[\frac{f_{i}(x)+x^{\mathrm{T}} z_{i}}{g_{i}(x)-x^{\mathrm{T}} v_{i}}-\frac{f_{i}(u)+u^{\mathrm{T}} z_{i}}{g_{i}(u)-u^{\mathrm{T}} v_{i}}\right] \\
& +\sum_{j=1}^{m} \mu_{j} \alpha_{j}^{2}(x, u)\left[h_{j}(x)+x^{\mathrm{T}} w_{j}-\left(h_{j}(u)+u^{\mathrm{T}} w_{j}\right)\right] \\
& \geq \eta^{\mathrm{T}}(x, u) \sum_{i=1}^{k} \lambda_{i}\left[\nabla\left(\frac{f_{i}(u)+u^{\mathrm{T}} z_{i}}{g_{i}(u)-u^{\mathrm{T}} v_{i}}\right)+\nabla_{p} \bar{H}_{i}(u, p)\right] \\
& +\eta^{\mathrm{T}}(x, u) \sum_{j=1}^{m} \mu_{j}\left[\nabla\left(h_{j}(u)+u^{\mathrm{T}} w_{j}\right)+\nabla_{p} G_{j}(u, p)\right] \\
& +\sum_{i=1}^{k} \lambda_{i} \bar{\beta}_{i}(x, u)\left[\bar{H}_{i}(u, p)-p^{\mathrm{T}} \nabla_{p} \bar{H}_{i}(u, p)\right] \\
& +\sum_{j=1}^{m} \mu_{j} \beta_{j}^{2}(x, u)\left[G_{j}(u, p)-p^{\mathrm{T}} \nabla_{p} G_{j}(u, p)\right] \\
& +\sum_{i=1}^{k} \lambda_{i} \bar{\rho}_{i}^{1}\left\|\bar{\theta}_{i}^{1}(x, u)\right\|^{2}+\sum_{j=1}^{m} \mu_{j} \rho_{j}^{2}\left\|\theta_{j}^{2}(x, u)\right\|^{2} .
\end{aligned}
$$

Using hypothesis 3) and (21), we get

$$
\begin{aligned}
& \sum_{i=1}^{k} \lambda_{i} \bar{\alpha}_{i}^{1}(x, u)\left[\frac{f_{i}(x)+x^{\mathrm{T}} z_{i}}{g_{i}(x)-x^{\mathrm{T}} v_{i}}-\frac{f_{i}(u)+u^{\mathrm{T}} z_{i}}{g_{i}(u)-u^{\mathrm{T}} v_{i}}\right] \\
& +\sum_{j=1}^{m} \mu_{j} \alpha_{j}^{2}(x, u)\left[h_{j}(x)+x^{\mathrm{T}} w_{j}-\left(h_{j}(u)+u^{\mathrm{T}} w_{j}\right)\right] \\
& \geq \sum_{i=1}^{k} \lambda_{i} \bar{\beta}_{i}^{1}(x, u)\left[\bar{H}_{i}(u, p)+p^{\mathrm{T}} \nabla_{p} \bar{H}_{i}(u, p)\right] \\
& +\sum_{j=1}^{m} \mu_{j} \beta_{j}^{2}(x, u)\left[G_{j}(u, p)-p^{\mathrm{T}} \nabla_{p} G_{j}(u, p)\right] .
\end{aligned}
$$

Finally, using hypothesis 4 ) and $x$ is feasible solution for (MFP), it follows that

$$
\begin{aligned}
\sum_{i=1}^{k} \lambda_{i}\left(\frac{f_{i}(x)+x^{\mathrm{T}} z_{i}}{g_{i}(x)-x^{\mathrm{T}} v_{i}}\right) \geq & \sum_{i=1}^{k} \lambda_{i}\left(\frac{f_{i}(u)+u^{\mathrm{T}} z_{i}}{g_{i}(u)-u^{\mathrm{T}} v_{i}}\right)+\sum_{j=1}^{m} \mu_{j}\left(h_{j}(u)+u^{\mathrm{T}} w_{j}\right) \\
& +\sum_{i=1}^{k} \lambda_{i}\left(\bar{H}_{i}(u, p)-p^{\mathrm{T}} \nabla_{p} \bar{H}_{i}(u, p)\right) \\
& +\sum_{j=1}^{m} \mu_{j}\left(G_{j}(u, p)-p^{\mathrm{T}} \nabla_{p} G_{j}(u, p)\right) .
\end{aligned}
$$

This contradicts Equation (24). Hence, the result.

Theorem 4.2. (Strong duality theorem). If $\bar{u} \in X^{0}$ is an efficient solution of (MFP) and the Slater's constraint qualification holds. Also, if for any $i=1,2, \cdots, k, j=1,2, \cdots, m$, 


$$
\bar{H}_{i}(\bar{u}, 0)=0, G_{j}(\bar{u}, 0)=0, \nabla_{p} \bar{H}_{i}(\bar{u}, 0)=0, \nabla_{p} G_{j}(\bar{u}, 0)=0,
$$

then there exist $\bar{\lambda} \in R^{k}, \bar{\mu} \in R^{m}, \bar{z}_{i} \in R^{n}, \bar{v}_{i} \in R^{n}$ and $\bar{w}_{j} \in R^{n}, i=1,2, \cdots, k, j=1,2, \cdots, m$, such that $(u, \bar{z}, \bar{v}, \bar{\mu}, \bar{\lambda}, \bar{w}, \bar{p}=0)$ is a feasible solution of (MFD) $)_{1}$ and the objective function values of (MFP) and $(\mathrm{MFD})_{1}$ are equal. Furthermore, if the hypotheses of Theorem 4.1 hold for all feasible solutions of (MFP) and (MFD) $)_{1}$ then, $(\bar{u}, \bar{z}, \bar{v}, \bar{\mu}, \bar{\lambda}, \bar{w}, \bar{p}=0)$ is an efficient solution of (MFD) $)_{1}$.

Proof. Since $\bar{u}$ is an efficient solution of (MFP) and the Slater's constraint qualification holds, then by Theorem 3.3, there exist

$\bar{\lambda} \in R^{k}, \bar{\mu} \in R^{m}, \bar{z}_{i} \in R^{n}, \bar{v}_{i} \in R^{n} \quad$ and $\quad \bar{w}_{j} \in R^{n}, i=1,2, \cdots, k, j=1,2, \cdots, m$, such that

$$
\begin{gathered}
\sum_{i=1}^{k} \bar{\lambda}_{i} \nabla\left(\frac{f_{i}(\bar{u})+\bar{u}^{\mathrm{T}} \bar{z}_{i}}{g_{i}(\bar{u})-\bar{u}^{\mathrm{T}} \bar{v}_{i}}\right)+\sum_{j=1}^{m} \bar{\mu}_{j} \nabla\left(h_{j}(\bar{u})+\bar{u}^{\mathrm{T}} \bar{w}_{j}\right)=0, \\
\sum_{j=1}^{m} \bar{\mu}_{j}\left(h_{j}(\bar{u})+\bar{u}^{\mathrm{T}} \bar{w}_{j}\right)=0, \\
\bar{u}^{\mathrm{T}} \bar{z}_{i}=S\left(\bar{u} \mid C_{i}\right), \bar{u}^{\mathrm{T}} \bar{v}_{i}=S\left(\bar{u} \mid D_{i}\right), \bar{u}^{\mathrm{T}} \bar{w}_{j}=S\left(\bar{u} \mid E_{j}\right), \\
\bar{z}_{i} \in C_{i}, \bar{v}_{i} \in D_{i}, \bar{w}_{j} \in E_{j}, \\
\bar{\lambda}_{i}>0, \sum_{i=1}^{k} \bar{\lambda}_{i}=1, \bar{\mu}_{j} \geq 0, i=1,2, \cdots, k, j=1,2, \cdots, m .
\end{gathered}
$$

Thus, $(\bar{u}, \bar{z}, \bar{v}, \bar{\mu}, \bar{\lambda}, \bar{w}, \bar{p}=0)$ is feasible for (MFD) $)_{1}$ and the objective function values of (MFP) and (MFD) ${ }_{1}$ are equal.

We now show that $(\bar{u}, \bar{z}, \bar{v}, \bar{\mu}, \bar{\lambda}, \bar{w}, \bar{p}=0)$ is an efficient solution of (MFD) $)_{1}$. If not, then there exists $\left(u^{\prime}, z^{\prime}, v^{\prime}, \mu^{\prime}, \lambda^{\prime}, w^{\prime}, p^{\prime}=0\right)$ of (MFD) $)_{1}$ such that

$$
\begin{aligned}
& \frac{f_{i}(\bar{u})+\bar{u}^{\mathrm{T}} \bar{z}_{i}}{g_{i}(\bar{u})-\bar{u}^{\mathrm{T}} \bar{v}_{i}}+\sum_{j=1}^{m} \bar{\mu}_{j}\left(h_{j}(\bar{u})+\bar{u}^{\mathrm{T}} \bar{w}_{j}\right) \\
& \leq \frac{f_{i}\left(u^{\prime}\right)+u^{\prime \mathrm{T}} z_{i}^{\prime}}{g_{i}\left(u^{\prime}\right)-u^{\prime \mathrm{T}} v_{i}^{\prime}}+\sum_{j=1}^{m} \mu_{j}^{\prime}\left(h_{j}\left(u^{\prime}\right)+u^{\prime \mathrm{T}} w_{j}^{\prime}\right), \text { for all } i=1,2, \cdots, k
\end{aligned}
$$

and

$$
\begin{aligned}
& \frac{f_{r}(\bar{u})+\bar{u}^{\mathrm{T}} \bar{z}_{r}}{g_{r}(\bar{u})-\bar{u}^{\mathrm{T}} \bar{v}_{r}}+\sum_{j=1}^{m} \bar{\mu}_{j}\left(h_{j}(\bar{u})+\bar{u}^{\mathrm{T}} \bar{w}_{j}\right) \\
& <\frac{f_{r}\left(u^{\prime}\right)+u^{\prime \mathrm{T}} z_{r}^{\prime}}{g_{r}\left(u^{\prime}\right)-u^{\prime \mathrm{T}} v_{r}^{\prime}}+\sum_{j=1}^{m} \mu_{j}^{\prime}\left(h_{j}\left(u^{\prime}\right)+u^{\prime \mathrm{T}} w_{j}^{\prime}\right), \text { for some } r=1,2, \cdots, k .
\end{aligned}
$$

By equation (31), we obtain

$$
\frac{f_{i}(\bar{u})+\bar{u}^{\mathrm{T}} \bar{z}_{i}}{g_{i}(\bar{u})-\bar{u}^{\mathrm{T}} \bar{v}_{i}} \leq \frac{f_{i}\left(u^{\prime}\right)+u^{\prime \mathrm{T}} z_{i}^{\prime}}{g_{i}\left(u^{\prime}\right)-u^{\prime \mathrm{T}} v_{i}^{\prime}}+\sum_{j=1}^{m} \mu_{j}^{\prime}\left(h_{j}\left(u^{\prime}\right)+u^{\prime \mathrm{T}} w_{j}^{\prime}\right), \text { for all } i=1,2, \cdots, k
$$

and

$$
\frac{f_{r}(\bar{u})+\bar{u}^{\mathrm{T}} \bar{z}_{r}}{g_{r}(\bar{u})-\bar{u}^{\mathrm{T}} \bar{v}_{r}}<\frac{f_{r}\left(u^{\prime}\right)+u^{\prime \mathrm{T}} z_{r}^{\prime}}{g_{r}\left(u^{\prime}\right)-u^{\prime \mathrm{T}} v_{r}^{\prime}}+\sum_{j=1}^{m} \mu_{j}^{\prime}\left(h_{j}\left(u^{\prime}\right)+u^{\prime \mathrm{T}} w_{j}^{\prime}\right), \text { for some } r=1,2, \cdots, k .
$$


This contradicts the Theorem 4.1. This complete the result.

Theorem 4.3. (Strict converse duality theorem). Let $\bar{x} \in X^{0}$ and $(\bar{u}, \bar{z}, \bar{v}, \bar{\mu}, \bar{\lambda}, \bar{w}, \bar{p}) \in Z^{0}$. Let

$$
\begin{aligned}
\sum_{i=1}^{k} \bar{\lambda}_{i}\left(\frac{f_{i}(\bar{x})+\bar{x}^{T} \bar{z}_{i}}{g_{i}(\bar{x})-\bar{x}^{T} \bar{v}_{i}}\right) \leq & \sum_{i=1}^{k} \bar{\lambda}_{i}\left(\frac{f_{i}(\bar{u})+\bar{u}^{\mathrm{T}} \bar{z}_{i}}{g_{i}(\bar{u})-\bar{u}^{\mathrm{T}} \bar{v}_{i}}\right)+\sum_{j=1}^{m} \bar{\mu}_{j}\left(h_{j}(\bar{u})+\bar{u}^{\mathrm{T}} \bar{w}_{j}\right) \\
& +\sum_{i=1}^{k} \bar{\lambda}_{i}\left(\bar{H}_{i}(\bar{u}, \bar{p})-\bar{p}^{\mathrm{T}} \nabla_{p} \bar{H}_{i}(\bar{u}, \bar{p})\right) \\
& +\sum_{j=1}^{m} \bar{\mu}_{j}\left(G_{j}(\bar{u}, \bar{p})-\bar{p}^{\mathrm{T}} \nabla_{p} G_{j}(\bar{u}, \bar{p})\right),
\end{aligned}
$$

1)

2) for any $i=1,2, \cdots, k,\left(f_{i}()+.(.)^{\mathrm{T}} \bar{z}_{i}\right)$ be strictly higher-order $\left(V, \alpha_{i}^{1}, \beta_{i}^{1}, \rho_{i}^{1}, \theta_{i}^{1}\right)$-invex at $\bar{u}$ with respect to $H_{i}(\bar{u}, \bar{p})$ and $-\left(g_{i}()+.(.)^{\mathrm{T}} \bar{v}_{i}\right)$ be higher-order $\left(V, \alpha_{i}^{1}, \beta_{i}^{1}, \rho_{i}^{1}, \theta_{i}^{1}\right)$-invex at $\bar{u}$ with respect to $H_{i}(\bar{u}, \bar{p})$,

3) for any $j=1,2, \cdots, m,\left(h_{j}()+.(.)^{\mathrm{T}} w_{j}\right)$ be higher-order $\left(V, \alpha_{j}^{2}, \beta_{j}^{2}, \rho_{j}^{2}, \theta_{j}^{2}\right)$-invex at $\bar{u}$ with respect to $G_{j}(\bar{u}, \bar{p})$,

4) $\sum_{i=1}^{k} \bar{\lambda}_{i} \bar{\rho}_{i}^{1}\left\|\bar{\theta}_{i}^{1}(\bar{x}, \bar{u})\right\|^{2}+\sum_{j=1}^{m} \bar{\mu}_{j} \rho_{j}^{2}\left\|\theta_{j}^{2}(\bar{x}, \bar{u})\right\|^{2} \geq 0$.

5) $\bar{\alpha}_{i}^{1}(\bar{x}, \bar{u})=\alpha_{j}^{2}(\bar{x}, \bar{u})=\beta_{i}^{1}(\bar{x}, \bar{u})=\beta_{j}^{2}(\bar{x}, \bar{u})=\alpha(\bar{x}, \bar{u}), \forall i=1,2, \cdots, k$, $j=1,2, \cdots, m$.

Then, $\bar{x}=\bar{u}$.

Proof. Using hypothesis 2) and Theorem 3.2, we have

$$
\begin{aligned}
& \bar{\alpha}_{i}^{1}(\bar{x}, \bar{u})\left[\frac{f_{i}(\bar{x})+\bar{x}^{\mathrm{T}} \bar{z}_{i}}{g_{i}(\bar{x})-\bar{x}^{\mathrm{T}} \bar{v}_{i}}-\frac{f_{i}(\bar{u})+\bar{u}^{\mathrm{T}} \bar{z}_{i}}{g_{i}(\bar{u})-\bar{u}^{\mathrm{T}} \bar{v}_{i}}\right] \\
& >\eta^{\mathrm{T}}(\bar{x}, \bar{u})\left[\nabla\left(\frac{f_{i}(\bar{u})+\bar{u}^{\mathrm{T}} \bar{z}_{i}}{g_{i}(\bar{u})-\bar{u}^{\mathrm{T}} \bar{v}_{i}}\right)+\nabla_{p} \bar{H}_{i}(\bar{u}, \bar{p})\right] \\
& +\bar{\beta}_{i}^{1}(\bar{x}, \bar{u})\left[\bar{H}_{i}(\bar{u}, \bar{p})-\bar{p}^{\mathrm{T}} \nabla_{p} \bar{H}_{i}(\bar{u}, \bar{p})\right]+\bar{\rho}_{i}^{1}\left\|\bar{\theta}_{i}^{1}(\bar{x}, \bar{u})\right\|^{2} .
\end{aligned}
$$

For any $j=1,2, \cdots, m,\left(h_{j}()+.(.)^{\mathrm{T}} w_{j}\right)$ is higher-order $\left(V, \alpha_{j}^{2}, \beta_{j}^{2}, \rho_{j}^{2}, \theta_{j}^{2}\right)$ -invex at $u$ with respect to $G_{j}(\bar{u}, \bar{p})$, we have

$$
\begin{aligned}
& \alpha_{j}^{2}(\bar{x}, \bar{u})\left[h_{j}(\bar{x})+\bar{x}^{\mathrm{T}} \bar{w}_{j}-\left(h_{j}(\bar{u})+\bar{u}^{\mathrm{T}} \bar{w}_{j}\right)\right] \\
& \geq \eta^{\mathrm{T}}(\bar{x}, \bar{u})\left[\nabla\left(h_{j}(\bar{u})+\bar{u}^{\mathrm{T}} \bar{w}_{j}\right)+\nabla_{p} G_{j}(\bar{u}, \bar{p})\right] \\
& +\beta_{j}^{2}(\bar{x}, \bar{u})\left[G_{j}(\bar{u}, \bar{p})-\bar{p}^{\mathrm{T}} \nabla_{p} G_{j}(\bar{u}, \bar{p})\right]+\rho_{j}^{2}\left\|\theta_{j}^{2}(\bar{x}, \bar{u})\right\|^{2} .
\end{aligned}
$$

Adding the two inequalities after multiplying (35) by $\bar{\lambda}_{i}$ and (36) by $\bar{\mu}_{j}$, we obtain

$$
\begin{aligned}
& \sum_{i=1}^{k} \bar{\lambda}_{i} \bar{\alpha}_{i}^{1}(\bar{x}, \bar{u})\left[\frac{f_{i}(\bar{x})+\bar{x}^{\mathrm{T}} \bar{z}_{i}}{g_{i}(\bar{x})-\bar{x}^{\mathrm{T}} \bar{v}_{i}}-\frac{f_{i}(\bar{u})+\bar{u}^{\mathrm{T}} \bar{z}_{i}}{g_{i}(\bar{u})-\bar{u}^{\mathrm{T}} \bar{v}_{i}}\right] \\
& +\sum_{j=1}^{m} \bar{\mu}_{j} \alpha_{j}^{2}(\bar{x}, \bar{u})\left[h_{j}(\bar{x})+\bar{x}^{\mathrm{T}} \bar{w}_{j}-\left(h_{j}(\bar{u})+\bar{u}^{\mathrm{T}} \bar{w}_{j}\right)\right] \\
& >\eta^{\mathrm{T}}(\bar{x}, \bar{u}) \sum_{i=1}^{k} \bar{\lambda}_{i}\left[\nabla\left(\frac{f_{i}(\bar{u})+\bar{u}^{\mathrm{T}} \bar{z}_{i}}{g_{i}(\bar{u})-\bar{u}^{\mathrm{T}} \bar{v}_{i}}\right)-\nabla_{p} H_{i}(\bar{u}, \bar{p})\right]
\end{aligned}
$$




$$
\begin{aligned}
& +\eta^{\mathrm{T}}(\bar{x}, \bar{u}) \sum_{j=1}^{m} \bar{\mu}_{j}\left[\nabla\left(h_{j}(\bar{u})+\bar{u}^{\mathrm{T}} \bar{w}_{j}\right)+\nabla_{p} G_{j}(\bar{u}, \bar{p})\right] \\
& +\sum_{i=1}^{k} \bar{\lambda}_{i} \bar{\beta}_{i}^{1}(\bar{x}, \bar{u})\left[\bar{H}_{i}(\bar{u}, \bar{p})-\bar{p}^{\mathrm{T}} \nabla_{p} \bar{H}_{i}(\bar{u}, \bar{p})\right] \\
& +\sum_{j=1}^{m} \bar{\mu}_{j} \beta_{j}^{2}(\bar{x}, \bar{u})\left[G_{j}(\bar{u}, \bar{p})-\bar{p}^{\mathrm{T}} \nabla_{p} G_{j}(\bar{u}, \bar{p})\right] \\
& +\sum_{i=1}^{k} \bar{\lambda}_{i} \bar{\rho}_{i}^{1}\left\|\bar{\theta}_{i}^{1}(\bar{x}, \bar{u})\right\|^{2}+\sum_{j=1}^{m} \bar{\mu}_{j} \rho_{j}^{2}\left\|\theta_{j}^{2}(\bar{x}, \bar{u})\right\|^{2}
\end{aligned}
$$

Using hypothesis 3 ) and (21), we get

$$
\begin{aligned}
& \sum_{i=1}^{k} \bar{\lambda}_{i} \bar{\alpha}_{i}^{1}(\bar{x}, \bar{u})\left[\frac{f_{i}(\bar{x})+\bar{x}^{\mathrm{T}} \bar{z}_{i}}{g_{i}(\bar{x})-\bar{x}^{\mathrm{T}} \bar{v}_{i}}-\frac{f_{i}(\bar{u})+\bar{u}^{\mathrm{T}} \bar{z}_{i}}{g_{i}(\bar{u})-\bar{u}^{\mathrm{T}} \bar{v}_{i}}\right] \\
& +\sum_{j=1}^{m} \bar{\mu}_{j} \alpha_{j}^{2}(\bar{x}, \bar{u})\left[h_{j}(\bar{x})+\bar{x}^{\mathrm{T}} \bar{w}_{j}-\left(h_{j}(\bar{u})+\bar{u}^{\mathrm{T}} \bar{w}_{j}\right)\right] \\
& >\sum_{i=1}^{k} \bar{\lambda}_{i} \bar{\beta}_{i}^{1}(\bar{x}, \bar{u})\left[\bar{H}_{i}(\bar{u}, \bar{p})-\bar{p}^{\mathrm{T}} \nabla_{p} \bar{H}_{i}(\bar{u}, \bar{p})\right] \\
& +\sum_{j=1}^{m} \bar{\mu}_{j} \beta_{j}^{2}(\bar{x}, \bar{u})\left[G_{j}(\bar{u}, \bar{p})-\bar{p}^{\mathrm{T}} \nabla_{p} G_{j}(\bar{u}, \bar{p})\right] .
\end{aligned}
$$

Finally, using hypothesis 4) and $\bar{x}$ is feasible solution for (MFP), it follows that

$$
\begin{aligned}
\sum_{i=1}^{k} \bar{\lambda}_{i}\left(\frac{f_{i}(\bar{x})+\bar{x}^{\mathrm{T}} \bar{z}_{i}}{g_{i}(\bar{x})-\bar{x}^{\mathrm{T}} \bar{v}_{i}}\right)> & \sum_{i=1}^{k} \bar{\lambda}_{i}\left(\frac{f_{i}(\bar{u})+\bar{u}^{\mathrm{T}} \bar{z}_{i}}{g_{i}(\bar{u})-\bar{u}^{\mathrm{T}} \bar{v}_{i}}\right)+\sum_{j=1}^{m} \bar{\mu}_{j}\left(h_{j}(\bar{u})+\bar{u}^{\mathrm{T}} \bar{w}_{j}\right) \\
& +\sum_{i=1}^{k} \bar{\lambda}_{i}\left(\bar{H}_{i}(\bar{u}, \bar{p})-\bar{p}^{\mathrm{T}} \nabla_{p} \bar{H}_{i}(\bar{u}, \bar{p})\right) \\
& +\sum_{j=1}^{m} \bar{\mu}_{j}\left(G_{j}(\bar{u}, \bar{p})-\bar{p}^{\mathrm{T}} \nabla_{p} G_{j}(\bar{u}, \bar{p})\right) .
\end{aligned}
$$

This contradicts the hypothesis 1$)$. Hence, the result.

\section{Duality Model-II}

Consider the following dual (MFD) $)_{2}$ of (MFP): (MFD) ${ }_{2}$ Maximize

$$
\left[\frac{f_{1}(u)+u^{\mathrm{T}} z_{1}}{g_{1}(u)-u^{\mathrm{T}} v_{1}}+\sum_{j=1}^{m} \mu_{j}\left(h_{j}(u)+u^{\mathrm{T}} w_{j}\right), \cdots, \frac{f_{k}(u)+u^{\mathrm{T}} z_{k}}{g_{k}(u)-u^{\mathrm{T}} v_{k}}+\sum_{j=1}^{m} \mu_{j}\left(h_{j}(u)+u^{\mathrm{T}} w_{j}\right)\right]
$$

subject to

$$
\begin{gathered}
\sum_{i=1}^{k} \lambda_{i} \nabla\left(\frac{f_{i}(u)+u^{\mathrm{T}} z_{i}}{g_{i}(u)-u^{\mathrm{T}} v_{i}}\right)+\sum_{j=1}^{m} \mu_{j} \nabla\left(h_{j}(u)+u^{\mathrm{T}} w_{j}\right) \\
+\sum_{i=1}^{k} \lambda_{i} \nabla_{p} H_{i}(u, p)+\sum_{j=1}^{m} \mu_{j} \nabla_{p} G_{j}(u, p)=0 \\
\sum_{i=1}^{k} \lambda_{i}\left(H_{i}(u, p)-p^{\mathrm{T}} \nabla_{p} H_{i}(u, p)\right)+\sum_{j=1}^{m} \mu_{j}\left(G_{j}(u, p)-p^{\mathrm{T}} \nabla_{p} G_{j}(u, p)\right) \geq 0 \\
z_{i} \in C_{i}, v_{i} \in D_{i}, w_{j} \in E_{j}, i=1,2, \cdots, k, j=1,2, \cdots, m
\end{gathered}
$$




$$
\mu_{j} \geq 0, \lambda_{i}>0, \sum_{i=1}^{k} \lambda_{i}=1, i=1,2, \cdots, k, j=1,2, \cdots, m .
$$

Let $P^{0}$ be the feasible solution for (MFD) $)_{2}$.

Theorem 5.1. (Weak duality theorem). Let $x \in X^{0}$ and $(u, z, v, y, \lambda, w, p) \in P^{0}$. Let for $i=1,2, \cdots, k, j=1,2, \cdots, m$,

1) $\left(\frac{f_{i}(.)+(.)^{\mathrm{T}} z_{i}}{g_{i}(.)-(.)^{\mathrm{T}} v_{i}}\right)$ be higher-order $\left(V, \alpha_{i}^{1}, \beta_{i}^{1}, \rho_{i}^{1}, \theta_{i}^{1}\right)$-invex at $u$ with respect to $H_{i}(u, p)$,

2) $\left(h_{j}()+.(.)^{\mathrm{T}} w_{j}\right)$ be higher-order $\left(V, \alpha_{j}^{2}, \beta_{j}^{2}, \rho_{j}^{2}, \theta_{j}^{2}\right)$-invex at $u$ with respect to $G_{j}(u, p)$,

3) $\sum_{i=1}^{k} \lambda_{i} \rho_{i}^{1}\left\|\theta_{i}^{1}(x, u)\right\|^{2}+\sum_{j=1}^{m} \mu_{j} \rho_{j}^{2}\left\|\theta_{j}^{2}(x, u)\right\|^{2} \geq 0$.

4) $\alpha_{i}^{1}(x, u)=\alpha_{j}^{2}(x, u)=\beta(x, u)=\beta_{j}^{2}(x, u)=\alpha(x, u)$.

Then the following cannot hold

$$
\frac{f_{i}(x)+S\left(x \mid C_{i}\right)}{g_{i}(x)-S\left(x \mid D_{i}\right)} \leq \frac{f_{i}(u)+u^{\mathrm{T}} z_{i}}{g_{i}(u)-u^{\mathrm{T}} v_{i}}+\sum_{j=1}^{m} \mu_{j}\left(h_{j}(u)+u^{\mathrm{T}} w_{j}\right), \forall i=1,2, \cdots, k
$$

and

$$
\begin{aligned}
& \frac{f_{r}(x)+S\left(x \mid C_{r}\right)}{g_{r}(x)-S\left(x \mid D_{r}\right)} \\
& <\frac{f_{r}(u)+u^{\mathrm{T}} z_{r}}{g_{r}(u)-u^{\mathrm{T}} v_{r}}+\sum_{j=1}^{m} \mu_{j}\left(h_{j}(u)+u^{\mathrm{T}} w_{j}\right), \text { for some } r=1,2, \cdots, k .
\end{aligned}
$$

Proof. The proof follows on the lines of Theorem 4.1.

Theorem 5.2 (Strong duality theorem). If $\bar{u} \in X^{0}$ is an efficient solution of (MFP) and the Slater's constraint qualification hold. Also, if for any

$$
\begin{aligned}
& i=1,2, \cdots, k, j=1,2, \cdots, m, \\
& H_{i}(\bar{u}, 0)=0, G_{j}(\bar{u}, 0)=0, \nabla_{p} H_{i}(\bar{u}, 0)=0, \nabla_{p} G_{j}(\bar{u}, 0)=0,
\end{aligned}
$$

then there exist $\bar{\lambda} \in R^{k}, \bar{\mu} \in R^{m}, \bar{z}_{i} \in R^{n}, \bar{v}_{i} \in R^{n}$ and $\bar{w}_{j} \in R^{n}, i=1,2, \cdots, k, j=1,2, \cdots, m$, such that $(u, \bar{z}, \bar{v}, \bar{\mu}, \bar{\lambda}, \bar{w}, \bar{p}=0) \quad$ is a feasible solution of (MFD) $)_{2}$ and the objective function values of (MFP) and $(\mathrm{MFD})_{2}$ are equal. Furthermore, if the conditions of Theorem 5.1 hold for all feasible solu- tions of (MFP) and (MFD) $)_{2}$ then, $(u, \bar{z}, \bar{v}, \bar{\mu}, \bar{\lambda}, \bar{w}, \bar{p}=0)$ is an efficient solution of (MFD) ${ }_{2}$.

Proof. The proof follows on the lines of Theorem 4.2.

Theorem 5.3. (Strict converse duality theorem). Let $\bar{x} \in X^{0}$ and $(\bar{u}, \bar{z}, \bar{v}, \bar{\mu}, \bar{\lambda}, \bar{w}, \bar{p}) \in P^{0}$. Let $i=1,2, \cdots, k, j=1,2, \cdots, m$,

1) $\sum_{i=1}^{k} \bar{\lambda}_{i}\left(\frac{f_{i}(\bar{x})+\bar{x}^{\mathrm{T}} \bar{z}_{i}}{g_{i}(\bar{x})-\bar{x}^{\mathrm{T}} \bar{v}_{i}}\right) \leq \sum_{i=1}^{k} \bar{\lambda}_{i}\left(\frac{f_{i}(\bar{u})+\bar{u}^{\mathrm{T}} \bar{z}_{i}}{g_{i}(\bar{u})-\bar{u}^{\mathrm{T}} \bar{v}_{i}}\right)+\sum_{j=1}^{m} \bar{\mu}_{j}\left(h_{j}(\bar{u})+\bar{u}^{\mathrm{T}} \bar{w}_{j}\right)$,

2) $\left(\frac{f_{i}(.)+(.)^{\mathrm{T}} \bar{z}_{i}}{g_{i}(.)-(.)^{\mathrm{T}} \bar{v}_{i}}\right)$ be strictly higher-order $\left(V, \alpha_{i}^{1}, \beta_{i}^{1}, \rho_{i}^{1}, \theta_{i}^{1}\right)$-invex at $\bar{u}$ with respect to $H_{i}(\bar{u}, \bar{p})$, 
3) $\left(h_{j}()+.(.)^{\mathrm{T}} w_{j}\right)$ be higher-order $\left(V, \alpha_{j}^{2}, \beta_{j}^{2}, \rho_{j}^{2}, \theta_{j}^{2}\right)$-invex at $\bar{u}$ with respect to $G_{j}(\bar{u}, \bar{p})$,

4) $\sum_{i=1}^{k} \bar{\lambda}_{i} \rho_{i}^{1}\left\|\theta_{i}^{1}(\bar{x}, \bar{u})\right\|^{2}+\sum_{j=1}^{m} \bar{\mu}_{j} \rho_{j}^{2}\left\|\theta_{j}^{2}(\bar{x}, \bar{u})\right\|^{2} \geq 0$.

5) $\alpha_{i}^{1}(\bar{x}, \bar{u})=\alpha_{j}^{2}(\bar{x}, \bar{u})=\beta_{i}^{1}(\bar{x}, \bar{u})=\beta_{j}^{2}(\bar{x}, \bar{u})=\alpha(\bar{x}, \bar{u})$.

Then, $\bar{x}=\bar{u}$.

Proof. The proof follows on the lines of Theorem 4.3.

\section{Duality Model-III}

Consider the following dual (MFD) ${ }_{3}$ of (MFP): (MFD) ${ }_{3}$ Maximize

$$
\begin{aligned}
& {\left[\frac{f_{1}(u)+u^{\mathrm{T}} z_{1}}{g_{1}(u)-u^{\mathrm{T}} v_{1}}+\left(\bar{H}_{1}(u, p)-p^{\mathrm{T}} \nabla_{p} \bar{H}_{1}(u, p)\right), \cdots,\right.} \\
& \left.\frac{f_{k}(u)+u^{\mathrm{T}} z_{k}}{g_{k}(u)-u^{\mathrm{T}} v_{k}}+\left(\bar{H}_{k}(u, p)-p^{\mathrm{T}} \nabla_{p} \bar{H}_{k}(u, p)\right)\right]
\end{aligned}
$$

subject to

$$
\begin{gathered}
\sum_{i=1}^{k} \lambda_{i} \nabla\left(\frac{f_{i}(u)+u^{\mathrm{T}} z_{i}}{g_{i}(u)-u^{\mathrm{T}} v_{i}}\right)+\sum_{j=1}^{m} \mu_{j} \nabla\left(h_{j}(u)+u^{\mathrm{T}} w_{j}\right) \\
+\sum_{i=1}^{k} \lambda_{i} \nabla_{p} \bar{H}_{i}(u, p)+\sum_{j=1}^{m} \mu_{j} \nabla_{p} G_{j}(u, p)=0, \\
\sum_{j=1}^{m} \mu_{j}\left[h_{j}(u)+u^{\mathrm{T}} w_{j}+G_{j}(u, p)-p^{\mathrm{T}} \nabla_{p} G_{j}(u, p)\right] \geq 0, \\
z_{i} \in C_{i}, v_{i} \in D_{i}, w_{j} \in E_{j}, i=1,2, \cdots, k, j=1,2, \cdots, m, \\
\mu_{j} \geq 0, \lambda_{i}>0, \sum_{i=1}^{k} \lambda_{i}=1, i=1,2, \cdots, k, j=1,2, \cdots, m .
\end{gathered}
$$

Let $S^{0}$ be feasible solution of (MFD) ${ }_{3}$.

Theorem 6.1. (Weak duality theorem). Let $x \in X^{0}$ and $(u, z, v, \mu, \lambda, w, p) \in S^{0}$. Let $i=1,2, \cdots, k, j=1,2, \cdots, m$,

1) $\left(f_{i}()+.(.)^{\mathrm{T}} z_{i}\right)$ and $-\left(g_{i}()-.(.)^{\mathrm{T}} v_{i}\right)$ be higher-order $\left(V, \alpha_{i}^{1}, \beta_{i}^{1}, \rho_{i}^{1}, \theta_{i}^{1}\right)$ -invex at $u$ with respect to $H_{i}(u, p)$,

2) $\left(h_{j}()+.(.)^{\mathrm{T}} w_{j}\right)$ be higher-order $\left(V, \alpha_{j}^{2}, \beta_{j}^{2}, \rho_{j}^{2}, \theta_{j}^{2}\right)$-invex at $u$ with respect to $G_{j}(u, p)$,

3) $\sum_{i=1}^{k} \lambda_{i} \bar{\rho}_{i}^{1}\left\|\bar{\theta}_{i}^{1}(x, u)\right\|^{2}+\sum_{j=1}^{m} \mu_{j} \rho_{j}^{2}\left\|\theta_{j}^{2}(x, u)\right\|^{2} \geq 0$.

4) $\bar{\alpha}_{i}^{1}(x, u)=\alpha_{j}^{2}(x, u)=\beta_{i}^{1}(x, u)=\beta_{j}^{2}(x, u)=\alpha(x, u)$,

where

$$
\begin{gathered}
\bar{\alpha}_{t}(x, u)=\left(\frac{g_{t}(x)-x^{\mathrm{T}} v_{t}}{g_{t}(u)-u^{\mathrm{T}} v_{t}}\right) \alpha_{t}(x, u), \quad \bar{\beta}_{t}(x, u)=\beta_{t}(x, u), \\
\bar{\theta}_{t}(x, u)=\theta_{t}(x, u)\left(\frac{1}{g_{t}(u)-u^{\mathrm{T}} v_{t}}+\frac{f_{t}(u)+u^{\mathrm{T}} z_{t}}{\left(g_{t}(u)-u^{\mathrm{T}} v_{t}\right)^{2}}\right)^{\frac{1}{2}}, \bar{\rho}_{t}(x, u)=\rho_{t}(x, u)
\end{gathered}
$$


and

$$
\bar{H}_{t}(u, p)=\left(\frac{1}{g_{t}(u)-u^{\mathrm{T}} v_{t}}+\frac{f_{t}(u)+u^{\mathrm{T}} z_{t}}{\left(g_{t}(u)-u^{\mathrm{T}} v_{t}\right)^{2}}\right) H_{t}(u, p) .
$$

Then, the following cannot hold

$$
\begin{aligned}
& \frac{f_{i}(x)+S\left(x \mid C_{i}\right)}{g_{i}(x)-S\left(x \mid D_{i}\right)} \\
& \leq \frac{f_{i}(u)+u^{\mathrm{T}} z_{i}}{g_{i}(u)-u^{\mathrm{T}} v_{i}}+\left(\bar{H}_{i}(u, p)-p^{\mathrm{T}} \nabla_{p} \bar{H}_{i}(u, p)\right), \text { for all } i=1,2, \cdots, k
\end{aligned}
$$

and

$$
\begin{aligned}
& \frac{f_{r}(x)+S\left(x \mid C_{r}\right)}{g_{r}(x)-S\left(x \mid D_{r}\right)} \\
& <\frac{f_{r}(u)+u^{\mathrm{T}} z_{r}}{g_{r}(u)-u^{\mathrm{T}} v_{r}}+\left(\bar{H}_{r}(u, p)-p^{T} \nabla_{p} \bar{H}_{r}(u, p)\right) \text {, for some } r=1,2, \cdots, k .
\end{aligned}
$$

Proof. The proof follows on the lines of Theorem 4.1.

Theorem 6.2. (Strong duality theorem). If $\bar{u} \in X^{0}$ is an efficient solution of (MFP) and let the Slater's constraint qualification be satisfied. Also, if for any $i=1,2, \cdots, k, j=1,2, \cdots, m$,

$$
\bar{H}_{i}(\bar{u}, 0)=0, G_{j}(\bar{u}, 0)=0, \nabla_{p} \bar{H}_{i}(\bar{u}, 0)=0, \nabla_{p} G_{j}(\bar{u}, 0)=0,
$$

then there exist $\bar{\lambda} \in R^{k}, \bar{\mu} \in R^{m}, \bar{z}_{i} \in R^{n}, \bar{v}_{i} \in R^{n}$ and $\bar{w}_{j} \in R^{n}, i=1,2, \cdots, k, j=1,2, \cdots, m$, such that $(u, \bar{z}, \bar{v}, \bar{\mu}, \bar{\lambda}, \bar{w}, \bar{p}=0) \quad$ is a feasible solution of (MFD) $)_{3}$ and the objective function values of (MFP) and $(\mathrm{MFD})_{3}$ are equal. Furthermore, if the conditions of Theorem 6.1 hold for all feasible solutions of (MFP) and (MFD) $)_{3}$ then, $(u, \bar{z}, \bar{v}, \bar{\mu}, \bar{\lambda}, \bar{w}, \bar{p}=0)$ is an efficient solution of (MFD) $)_{3}$.

Proof. The proof follows on the lines of Theorem 4.2.

Theorem 6.3. (Strict converse duality theorem). Let $\bar{x} \in X^{0}$ and $(\bar{u}, \bar{z}, \bar{v}, \bar{\mu}, \bar{\lambda}, \bar{w}, \bar{p})$ be feasible for (MFD) ${ }_{3}$. Suppose that:

1)

$$
\sum_{i=1}^{k} \bar{\lambda}_{i}\left(\frac{f_{i}(\bar{x})+\bar{x}^{\mathrm{T}} \bar{z}_{i}}{g_{i}(\bar{x})-\bar{x}^{\mathrm{T}} \bar{v}_{i}}\right) \leq \sum_{i=1}^{k} \bar{\lambda}_{i}\left(\frac{f_{i}(\bar{u})+\bar{u}^{\mathrm{T}} \bar{z}_{i}}{g_{i}(\bar{u})-\bar{u}^{\mathrm{T}} \bar{v}_{i}}\right)+\sum_{i=1}^{k} \bar{\lambda}_{i}\left(\bar{H}_{i}(\bar{x}, \bar{u})-\bar{p}^{T} \nabla_{p} \bar{H}(\bar{x}, \bar{u})\right),
$$

2) for any $i=1,2, \cdots, k,\left(f_{i}()+.(.)^{\mathrm{T}} \bar{z}_{i}\right)$ be strictly higher-order $\left(V, \alpha_{i}^{1}, \beta_{i}^{1}, \rho_{i}^{1}, \theta_{i}^{1}\right)$-invex at $\bar{u}$ with respect to $H_{i}(\bar{u}, \bar{p})$ and $-\left(g_{i}()+.(.)^{\mathrm{T}} \bar{v}_{i}\right)$ be higher-order $\left(V, \alpha_{i}^{1}, \beta_{i}^{1}, \rho_{i}^{1}, \theta_{i}^{1}\right)$-invex at $\bar{u}$ with respect to $H_{i}(\bar{u}, \bar{p})$,

3) for any $j=1,2, \cdots, m,\left(h_{j}()+.(.)^{\mathrm{T}} w_{j}\right)$ is higher-order $\left(V, \alpha_{j}^{2}, \beta_{j}^{2}, \rho_{j}^{2}, \theta_{j}^{2}\right)$ -invex at $\bar{u}$ with respect to $G_{j}(\bar{u}, \bar{p})$,

$$
\begin{aligned}
& \text { 4) } \sum_{i=1}^{k} \bar{\lambda}_{i} \bar{\rho}_{i}^{1}\left\|\bar{\theta}_{i}^{1}(\bar{x}, \bar{u})\right\|^{2}+\sum_{j=1}^{m} \bar{\mu}_{j} \rho_{j}^{2}\left\|\theta_{j}^{2}(\bar{x}, \bar{u})\right\|^{2} \geq 0 . \\
& \text { 5) } \bar{\alpha}_{i}^{1}(\bar{x}, \bar{u})=\alpha_{j}^{2}(\bar{x}, \bar{u})=\beta_{i}^{1}(\bar{x}, \bar{u})=\beta_{j}^{2}(\bar{x}, \bar{u})=\alpha(\bar{x}, \bar{u}), \forall i=1,2, \cdots, k, \\
& j=1,2, \cdots, m .
\end{aligned}
$$


Then, $\bar{x}=\bar{u}$.

Proof. The proof follows on the lines of Theorem 4.3.

\section{Conclusion}

In this paper, we consider a class of non differentiable multiobjective fractional programming (MFP) with higher-order terms in which each numerator and denominator of the objective function contains the support function of a compact convex set. Furthermore, various duality models for higher-order have been formulated for (MFP) and appropriate duality relations have been obtained under higher-order $(V, \alpha, \beta, \rho, d)$-invexity assumptions.

\section{Acknowledgements}

The second author is grateful to the Ministry of Human Resource and Development, India for financial support, to carry this work.

\section{References}

[1] Mangasarian, O.L. (1975) Second and Higher-Order Duality in Nonlinear Programming. Journal of Mathematical Analysis and Applications, 51, 607-620. https://doi.org/10.1016/0022-247X(75)90111-0

[2] Egudo, R.R. (1988) Multiobjective Fractional Duality. Bulletin of the Australian Mathematical Society, 37, 367-378. https://doi.org/10.1017/S0004972700026988

[3] Gulati, T.R. and Agarwal, D. (2008) Optimality and Duality in Nondifferentiable Multiobjective Mathematical Programming Involving Higher Order $(F, \alpha, \rho, d)$ Type I Functions. Journal of Applied Mathematics and Computing, 27, 345-364. https://doi.org/10.1007/s12190-008-0069-9

[4] Chen, X. (2004) Higher-Order Symmetric Duality in Nondifferentiable Multiobjective Programming Problems. Journal of Mathematical Analysis and Applications, 290, 423-435. https://doi.org/10.1016/j.jmaa.2003.10.004

[5] Suneja, S.K., Srivastava, M.K. and Bhatia, M. (2008) Higher Order Duality in Multiobjective Fractional Programming with Support Functions. Journal of Mathematical Analysis and Applications, 347, 8-17. https://doi.org/10.1016/j.jmaa.2008.05.056

[6] Dubey, R. and Gupta, S.K. (2016) Duality for a Nondifferentiable Multiobjective Higher-Order Symmetric Fractional Programming Problems with Cone Constraints. Journal of Nonlinear Analysis and Optimization: Theory and Applications, 7, 1-15.

[7] Gupta, S.K., Dubey, R. and Debnath, I.P. (2017) Second-Order Multiobjective Programming Problems and Symmetric Duality Relations with $G_{f}$-Bonvexity. OPSEARCH, 54, 365-387. https://doi.org/10.1007/s12597-016-0280-7

[8] Kuk, H., Lee, G.M. and Kim, D.S. (1998) Nonsmooth Multiobjective Programs with V- $\rho$-Invexity. Indian Journal of Pure and Applied Mathematics, 29, 405-412.

[9] Jeyakumar, V. and Mond, B. (1992) On Generalised Convex Mathematical Programming. Journal of the Australian Mathematical Society Series B, 34, 43-53. https://doi.org/10.1017/S0334270000007372

[10] Pandey, S. (1991) Duality for Multiobjective Fractional Programming Involving Generalized $\eta$-Bonvex Functions. OPSEARCH, 28, 31-43.

[11] Gulati, T.R. and Geeta (2011) Duality in Nondifferentiable Multiobjective Frac- 
tional Programming Problem with Generalized Invexity. Journal of Applied Mathematics and Computing, 35, 103-118. https://doi.org/10.1007/s12190-009-0345-3

[12] Gupta, S.K., Kailey, N. and Sharma, M.K. (2010) Multiobjective Second-Order Nondifferentiable Symmetric Duality Involving $(F, \alpha, \rho, d)$-Convex Function. Journal of Applied Mathematics and Informatics, 28, 1395-1408.

[13] Dubey, R., Gupta, S.K. and Khan, M.A. (2015) Optimality and Duality Results for a Nondifferentiable Multiobjective Fractional Programming Problem. Journal of Inequalities and Applications, 354. https://doi.org/10.1186/s13660-015-0876-0

[14] Reddy, L.V. and Mukherjee, R.N. (1999) Some Results on Mathematical Programming with Generalized Ratio Invexity. Journal of Mathematical Analysis and Applications, 240, 299-310. https://doi.org/10.1006/jmaa.1999.6334 